\title{
Non-traumatic Musculoskeletal Diseases in Children
}

\author{
Rutger A. J. Nievelstein
}

\section{Learning Objectives}

- To gain knowledge about the differences between the pediatric and adult musculoskeletal system.

- To learn how these differences will result in differences in clinical and radiological presentation of musculoskeletal diseases in children.

- To discuss the optimal imaging strategies for specific disease entities in the pediatric musculoskeletal system.

\subsection{Introduction}

The pediatric musculoskeletal system differs from the adult musculoskeletal system in many ways, which accounts for many unique features in pediatric musculoskeletal imaging. These unique features include:

- Anatomical differences: such as growth plates with surrounding epiphyses and metaphyses and differences in vascular supply (the Haversian canals in pediatric bones are relatively larger and more extensive compared to adults).

- Physiological differences: such as increased metabolism at the metaphyses related to growth and physiological subperiosteal new bone formation in infants.

- Psychological differences: in case of pain, young children experience the uncomfortable sensation, but they are not worried by it and do not seek medical attention themselves. Instead they try to relief the pain by crying, limp-

\footnotetext{
R. A. J. Nievelstein ( $\bowtie)$

Department of Radiology \& Nuclear Medicine, Division Imaging

\& Oncology, Wilhelmina Children's Hospital - University Medical Center Utrecht \& Princess Maxima Center of Pediatric Oncology,

Utrecht, Utrecht, The Netherlands

e-mail: r.a.j.nievelstein@umcutrecht.nl
}

ing, or disuse, and this is what is noted by the parents. Moreover, young children usually cannot give an appropriate history. Finally, "referred pain" is more often seen in children than in adults: for example, pain in the knee in a young child may be the result of referred pain from the distal tibia (e.g., a toddler fracture) or from the hip (e.g., in Perthes disease).

In addition to these differences, several pathologies in the musculoskeletal system are unique for the pediatric age and do not or seldomly occur in adults (except for some late sequelae). These include congenital and hereditary disorders, many inborn errors of metabolism and skeletal dysplasias, and some subtypes of bone and soft tissue tumors.

These differences between the pediatric and adult musculoskeletal system can result in differences in clinical and radiological presentation. Therefore, radiologists who deal with children require specific knowledge and skills, including knowledge of the (combination of) imaging modalities of first choice to answer the clinical question and optimization of the imaging techniques used (while limiting the risks of ionizing radiation, sedation, and unnecessary biopsies). In this chapter on non-traumatic pediatric musculoskeletal diseases, we will focus on (a) hip pathologies, (b) infectious and inflammatory diseases, and (c) bone and soft tissue tumors.

\subsection{Hip Pathologies [1-4]}

\subsubsection{Developmental Dysplasia of the Hip (DDH) [3-7]}

Developmental dysplasia of the hip (DDH), also known as congenital hip dysplasia (CHD), is one of the commonest musculoskeletal pathologies in the newborn. It is a developmental (and not a congenital!) disease of the hip as newborns with normal hips at birth can be affected later during infancy. Therefore, CHD is a misnomer and should be avoided. 
DDH occurs in approximately 1:1000 live births with a female predominance. The left hip is more commonly involved than the right hip, and it can occur bilaterally. Risk factors include breech position during pregnancy (girls $12 \%$, boys $2.6 \%$ ) and a positive family history (girls $4.4 \%$, boys $0.9 \%)$. Clubfoot has been thought to be a risk factor but this no longer holds true.

Ultrasound (US) is the imaging technique of first choice in infants younger than 6-9 months of age with suspicion of DDH. After this age the physiological ossification of the femoral epiphyses usually obscures an adequate overview of the entire hip joint and a pelvic X-ray is preferred. With US we are looking at the same anatomic structures as on a pelvic $\mathrm{X}$-ray, with the advantage of also displaying the cartilaginous structures of the hip joint (Fig. 20.1). DDH on US is classified according to the Graf classification in types I-IV based on the morphology of the iliac bone, including the shape of the acetabulum, the bony and cartilaginous acetabular rim, labrum, and position of the femoral head (Table 20.1). It has been shown that US can also be used for guidance and follow-up of manually as well as operatively reduced dislocated dysplastic hips.

\subsubsection{Transient Synovitis (Coxitis Fugax) [1, 2]}

Transient synovitis of the hip is an aseptic inflammation. It is the most common cause of hip pain or limping in children under the age of 10 years and is thought to have a postviral etiology. Although limping or hip pain is usually the only complaint at presentation, affected children might be mildly ill or recently have suffered from a low-grade upper respiratory tract infection. Transient synovitis is a self-limiting disease, usually only requiring rest and analgesics as treatment. Although imaging is not strictly indicated, an US is often performed to confirm the diagnosis and/or rule out other causes. The only finding on US is a (small) joint effusion. Pelvic X-ray is only indicated when other differential diagnoses are considered. In case of a sick child, a septic arthritis should always be considered.

\subsubsection{Legg-Calvé-Perthes Disease (Perthes Disease) $[2-4,8-10]$}

Perthes disease is an idiopathic avascular necrosis of the proximal femoral epiphysis. It typically occurs between the

Table 20.1 Graf's classification for developmental dysplasia of the hip (short version) [4, 5]

\begin{tabular}{l|l|l|} 
Type & $\alpha$ angle $^{\mathrm{a}}$ & Additional features \\
\hline Type I & $>60^{\circ}$ & \\
\hline Type IIa & $50-59^{\circ}$ & Appropriate for age \\
\hline Type IIb & $50-59^{\circ}$ & Inappropriate for age \\
\hline Type IIc & $43-49^{\circ}$ & \\
\hline Type D & $43-49^{\circ}$ & Decentering hip \\
\hline Type III & $<43^{\circ}$ & Eccentric hip \\
\hline Type IV & $<43^{\circ}$ & Inverted labrum \\
\hline
\end{tabular}

aThe $\alpha$ angle is a measurement of the bony roof of the acetabulum on ultrasound
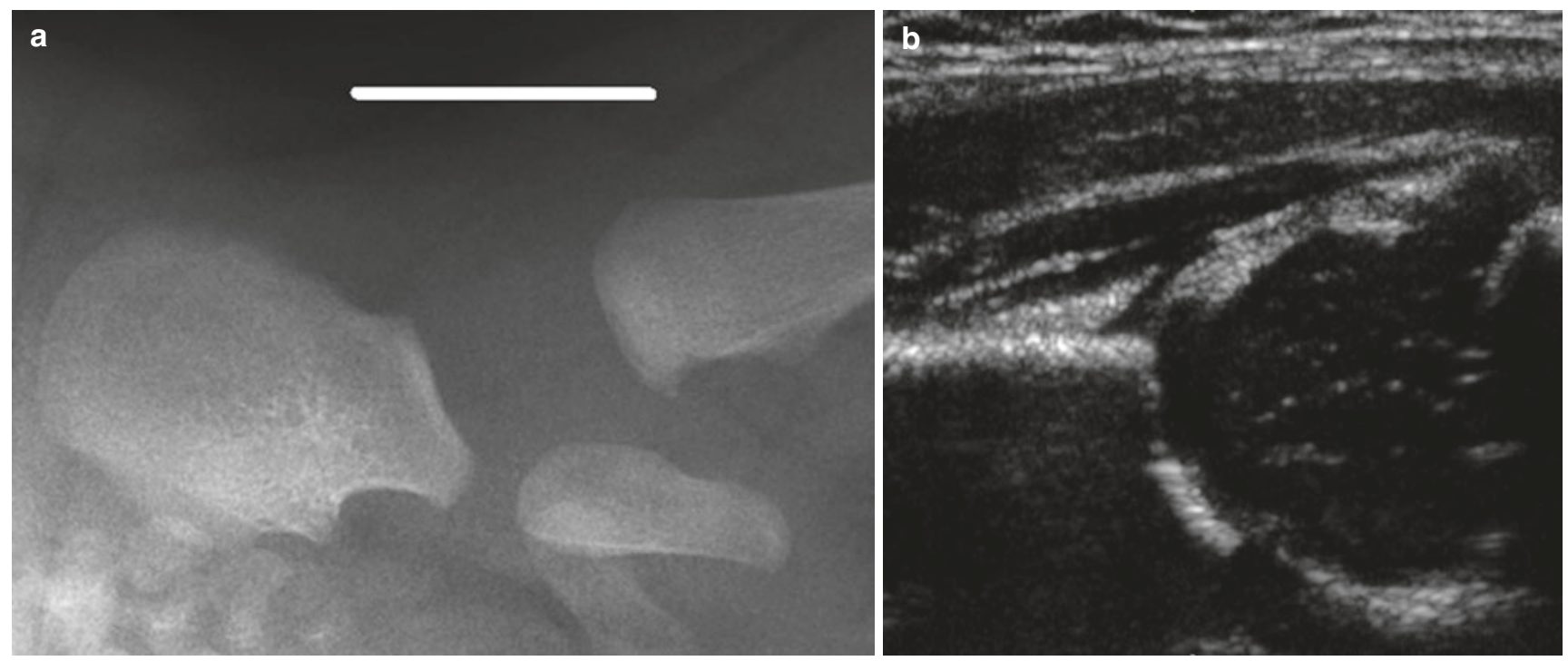

Fig. 20.1 Illustration of how the ultrasound (US) of the hip in DDH is performed (a) and displayed (b). The white horizontal line projected on the X-ray of the pelvis (a) represents the position of the US transducer. (Courtesy of S.G.F. Robben, MUMC, Maastricht, the Netherlands) 

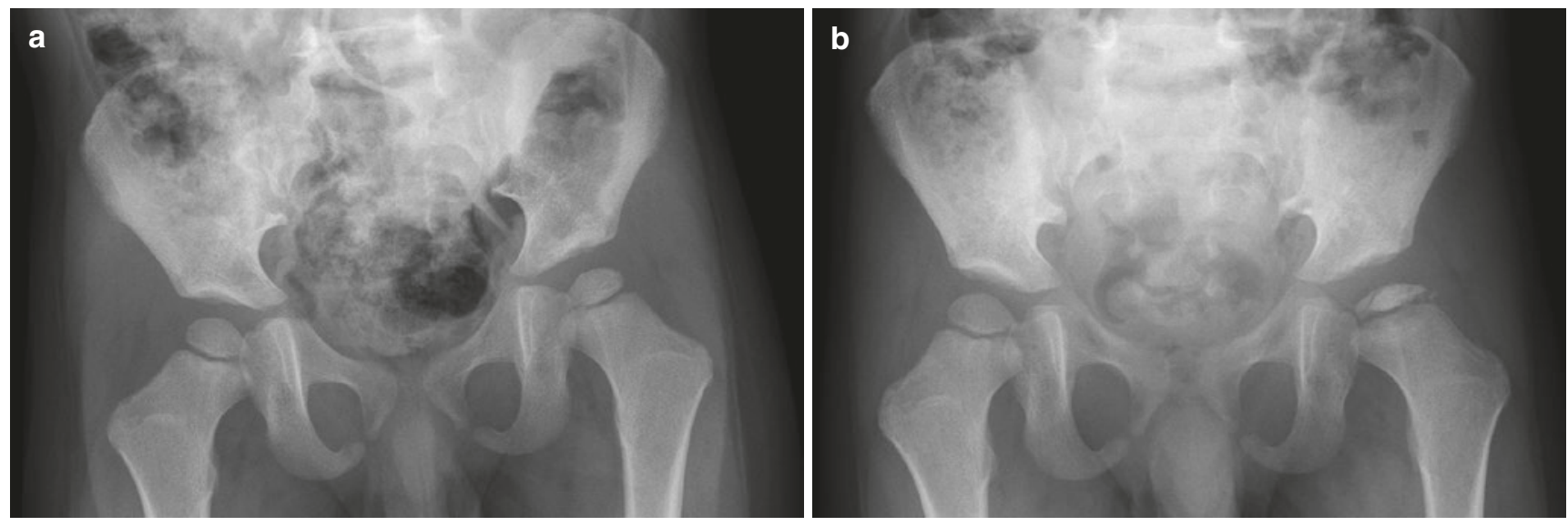

Fig. 20.2 Pelvic X-rays in Perthes disease. (a) In the early phase, only subtle flattening and sclerosis of the epiphysis of the left hip is seen. (b) A few months later progressive flattening with fragmentation and sclerosis of the epiphysis has developed

age of 5 and 8 years (range 3-12 years) with a male predominance. Although usually unilateral, Perthes disease can occur bilaterally. In bilateral cases its presentation is usually asymmetric in contrast to epiphyseal skeletal dysplasias. On US a joint effusion can be the only sign in early stages. Other US findings include collapse and fragmentation of the epiphysis and atrophy of the quadriceps muscles (due to disuse). Pelvic X-ray is, however, the most commonly used imaging modality in (suspicion of) Perthes disease but might be normal or show only subtle flattening of the epiphysis in early stages (Fig. 20.2). In later stages, progressive flattening and collapse, sclerosis, and subchondral fractures can develop, best appreciated on the frog-leg lateral view. Furthermore, metaphyseal lucencies can occur. During the healing phase, Perthes disease often results in a broad femoral head and collum (also called coxa magna) with a secondary enlarged and somewhat shallow acetabulum. Magnetic resonance imaging (MRI) can be performed but is usually only indicated in complex cases in which surgical reconstruction is considered. In the early phase, MRI will show bone marrow edema in the proximal femoral epiphysis on T2-weighted images with loss of high (fatty) bone marrow signal on T1-weighted images. A subchondral (and sometimes radiographically occult) fracture can be detected on MRI as a double rim sign on T2-weighted images with fat saturation. Furthermore, joint effusion may be present and the cartilage may become hypertrophic. Although treatment is usually conservative and symptomatic, sometimes surgical reconstruction is indicated (e.g., Salter osteotomy) to prevent development of early osteoarthritis. Besides epiphyseal skeletal dysplasias, the most important differential diagnostic consideration should include secondary avascular necrosis (due to corticosteroid therapy, sickle cell anemia, SLE or as complication of hip dysplasia treatment). In the latter, the clinical information will usually help to distinguish it from Perthes disease.

\subsubsection{Slipped Capital Femoral Epiphysis (SCFE) $[2-4,9]$}

Slipped capital femoral epiphysis (SCFE) is an idiopathic Salter-Harris type 1 epiphysiolysis of the proximal femoral epiphysis. It more commonly affects boys and obese children with a typical age at presentation between 12 and 15 years. In approximately one-third of cases, SCFE occurs bilaterally. On imaging the epiphysis is displaced posteriorly and slightly medially, which is best appreciated on the frog-leg lateral view (Fig. 20.3). To prevent further slippage, SCFE is treated by surgical fixation. Avascular necrosis of the femoral head is a potential complication.

\section{Key Point}

- Ultrasound (US) and conventional radiography (CR) are the imaging modalities of first choice in pediatric patients with suspicion of hip pathology.

\subsection{Infectious and Inflammatory Diseases}

\subsubsection{Osteomyelitis [11-16]}

Infections of the bone and bone marrow are rare in children with a reported incidence of approximately 1:5000 children. More than half of the cases occur in children under 5 years of age, and boys are twice as often affected than girls. The lower limb is involved in over $70 \%$ of cases with the knee as most common location. It is usually mono-ostotic at presentation but can be polyostotic, particularly in neonates (22\%) and infants (6.8\%). Children with hemoglobinopathies (e.g., sickle cell disease) and immunodeficiencies are at increased risk of developing osteomyelitis. 
Osteomyelitis usually develops via hematogenous spread, often after a minor trauma. Other routes of infection include direct inoculation and local extension from contiguous soft tissue infection. The most common organism involved is Staphylococcus aureus. Less frequently, $\beta$-hemolytic Streptococcus, Streptococcus pneumonia, Escherichia coli, Pseudomonas aeruginosa, and Haemophilus influenzae are cultured from blood or aspirates, although the incidence of Haemophilus influenzae osteomyelitis has decreased dramatically since the introduction of HIB vaccination. In neonates, Enterobacteria and Candida albicans can also cause osteomyelitis, and in infants Kingella kingae is increasingly cultured. It should be mentioned, however, that blood cultures are only positive in $30-50 \%$ of cases.

The presentation of osteomyelitis in children is agedependent and related to the developing skeleton. In infants diaphysial vessels still penetrate the growth plate to reach the

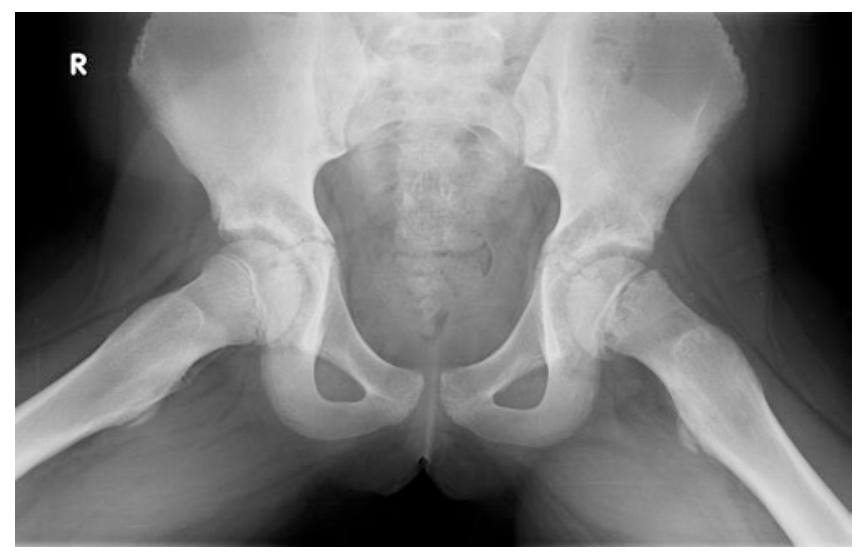

Fig. 20.3 Frog-leg view of the pelvis demonstrating the posterior and slightly medially displaced epiphysis of the left hip in slipped capital femoral epiphysis (SCFE)

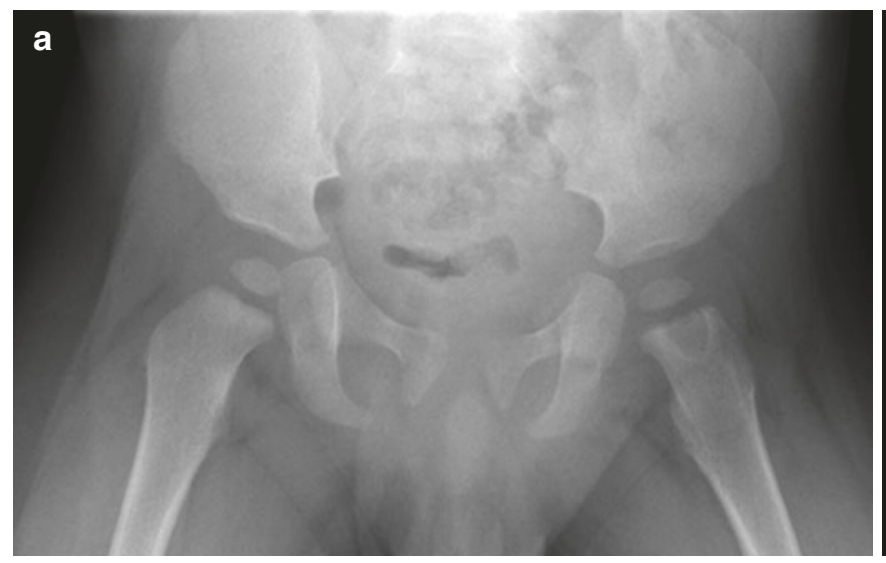

Fig. 20.4 Osteomyelitis in a 1-year-old boy with fever and left limb pain. (a) Pelvic X-ray demonstrating a metaphyseal located radiolucency suspicious of osteomyelitis. (b) Coronal T2 STIR-weighted MR epiphysis, facilitating epiphyseal and joint infections in this age group. In contrast, in older children, the growth plate constitutes a barrier for the diaphysial vessels. These vessels terminate at the metaphysis in slow flow venous sinusoidal lakes, predisposing the metaphysis as the starting point for acute hematogenous osteomyelitis. Furthermore, because the periosteum is less firmly attached to the cortex in infants and children than in adults, elevation will be more pronounced in childhood osteomyelitis. Sequestration is infrequently seen and rare in neonates.

When osteomyelitis is suspected, conventional radiography (CR) is usually the modality of first choice (Fig. 20.4a). In the early phase, soft tissue swelling is often the only sign detected, as bone destruction and periosteal reaction become obvious only 7-10 days after the onset of disease and periosteal thickening is a late finding. With US, cortical defects and subperiosteal as well as juxtacortical soft tissue swelling and abscesses can be easily detected. The detection of these abscesses is important as these patients often require US-guided or surgical drainage instead of antibiotics only. Although computed tomography (CT) will demonstrate osseous changes earlier than $\mathrm{CR}$, it is not a preferred technique because of its higher radiation dose. However, CT is superior to other imaging modalities (including MRI) in visualizing osseous destruction, gas in the bone, and bone sequestration. Because of its excellent soft tissue contrast, MRI is an invaluable imaging technique for more clearly demonstrating intra- and extraosseous extension of the osteomyelitis, particularly in complex cases (Fig. 20.4b). Imaging characteristics include ill-defined low T1 and high T2 signal intensity of the bone marrow, periosteal thickening, poorly defined soft tissue planes, lack of cortical thickening, and poor interface between normal and abnormal marrow. Another imaging sign that can be seen are the so-called "fatty globules" in the infected bone marrow best seen on T1-weighted sequences.

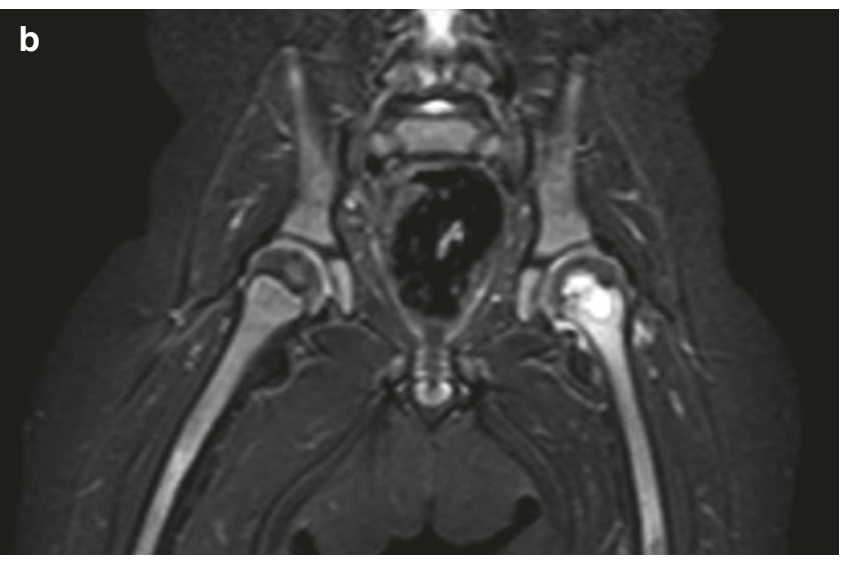

image of the pelvis and hip nicely illustrating the involvement of both the metaphyses and epiphysis of the left hip with surrounding bone marrow edema, joint effusion, and only minor soft tissue changes 
The use of gadolinium is mandatory and increases the diagnostic confidence as well as the detection of (small) abscesses and draining sinuses. Nuclear medicine (including 99mTcmethylene diphosphonate scintigraphy (99mTc-MDP scintigraphy) or $18 \mathrm{~F}$-fluorodeoxyglucose positron emission tomography/CT (18F-FDG-PET/CT)) can be used for the detection of osteomyelitis but at the expense of higher radiation doses. These techniques are usually reserved for those cases where the site of infection is unclear or if MRI is not possible or available (e.g., because of sedation/anesthesia).

\subsection{2 (Spondylo)discitis [17]}

Spondylitis or (spondylo)discitis in children is usually a lowgrade infection of the vertebral body and/or intervertebral disc. The average age at diagnosis is $2-8$ years, which is explained by the fact that the vascularization of the vertebral bodies in young children is made up of vessels across the cartilaginous vertebral plate and into the ring. After the age of 8 years, these vessels disappear, but a rich anastomotic network of vessels remains in the periphery of the disc until adolescence. (Spondylo)discitis in children mainly involves the lumbar and lumbosacral spine. Many organisms can cause spondylodiscitis, but again positive cultures are only seen in up to $50 \%$ of cases. In the early stages of the disease, CR has low sensitivity and specificity. The first radiological signs include intervertebral disc space narrowing with indistinct endplates on either side and eventually destruction of the endplates, but these signs can occur for the first time up to 2-8 weeks after the onset of symptoms. MRI is the imaging modality of choice in case of (suspected) (spondylo)discitis, demonstrating signal intensity abnormalities in the intervertebral disc and adjacent vertebral bodies. Paravertebral soft tissue extension (including abscesses) can also be easily seen.

\subsubsection{Septic Arthritis [14, 16, 18]}

Intra-articular infections in children are most commonly caused by hematogenous spread of microorganisms into the joints. However, in infants direct extension into the joint space from adjacent osteomyelitis can also cause septic arthritis, due to the diaphysial vessels that still penetrate the growth plate to reach the epiphysis. The hip joint is the most frequent location, followed by the knee, shoulder, and elbow. Early diagnosis is mandatory to prevent cartilage destruction, joint deformity, growth disturbance, and eventually premature arthrosis. As in osteomyelitis, the most common etiologic organism is Staphylococcus aureus. Other etiologic organisms include group A Streptococcus and Streptococcus pneumoniae, group B Streptococcus and Escherichia coli (in neonates), and Neisseria gonorrhoeae in adolescents.
As many children do not present with an obvious clinical picture, imaging is important to detect septic arthritis and give additional information of the suspected joint. CR has low sensitivity and specificity for septic arthritis, can be normal, or can demonstrate joint space widening with adjacent soft tissue swelling. US is a very sensitive technique for the detection of joint effusion, and small amounts up to $1 \mathrm{ml}$ can be detected. However, the specificity toward the diagnosis is poor as neither the amount nor the echogenicity of the effusion can distinguish infectious from noninfectious effusion. On the other hand, the absence of joint effusion virtually excludes septic arthritis. Again, MRI is very sensitive for the detection of joint effusions and synovial disease. In the early stage of the infection, the joint effusion tends to have a nonspecific T2 high signal intensity, whereas in later stages, it tends to have a more intermediate and heterogeneous signal intensity. Fat suppression sequences after intravenous gadolinium injection will show synovial enhancement. Moreover, MRI can demonstrate cartilage destruction and adjacent soft tissue involvement.

\section{Key Point}

- The clinical and radiological presentation of osteomyelitis, (spondylo)discitis, and septic arthritis in children is age-dependent and related to the developing skeleton.

\subsubsection{Soft Tissue Infections $[19,20]$}

This includes cellulitis, soft tissue abscesses, pyomyositis, and necrotizing fasciitis. In children there is a predilection of these types of infections for the extremities. The most common etiologic organisms are Staphylococcus aureus and Streptococcus pyogenes. The preferred imaging techniques to investigate these infections include US (including color Doppler imaging) and MRI (including contrast-enhanced and diffusion-weighted imaging) because of their high spatial resolution and excellent soft tissue contrast. A detailed discussion falls beyond the scope of this chapter and the interested reader is referred to the literature.

\subsubsection{Chronic Recurrent Multifocal Osteomyelitis (CRMO) [21-23]}

Chronic recurrent multifocal osteomyelitis (CRMO) or chronic nonbacterial osteomyelitis is a rare and poorly understood disease that primarily occurs in late childhood and adolescence. It is believed to be the pediatric variant of SAPHO syndrome, which is a combination of synovitis, 
acne, pustulosis, hyperostosis, and osteitis. The exact etiology is unknown although it is thought to be an autoimmune disorder. CRMO most commonly affects the lower limbs followed by the pelvis, spine, and anterior chest wall (including the claviculae). MRI is the imaging modality of choice showing nonspecific multifocal and often symmetric bone marrow signal intensity changes ( $\mathrm{T} 1$ hypointense and T2 hyperintense), usually located in the metaphysis of the long bones, and with minimal soft tissue involvement. MRI is especially useful to demonstrate the extent of the disease, detecting asymptomatic sites in up to $20 \%$ of cases. CR has low sensitivity and specificity but may demonstrate focal lysis at the involved sites, reactive hyperostosis, and periosteal reaction.

\subsubsection{Juvenile Idiopathic Arthritis (JIA)} $[18,24-27]$

Juvenile idiopathic arthritis (JIA) encompasses seven subtypes of (aseptic) arthritis with onset before the age of 16 years and persistence of symptoms for more than 6 weeks (Table 20.2). Its prevalence varies between 0.07 and 4.01 per 1000 children. JIA most commonly involves the knee, followed by the ankle, wrist, hand, and others. The etiology and pathophysiology of JIA are still not completely understood. Early manifestations of JIA include synovial inflammation, causing periarticular demineralization and joint effusion. Ongoing inflammation will result in synoviocyte prolifera- tion, increased metabolic requirements, and neovascularization which causes pannus formation. Furthermore, it is thought that autoantibody depositions and degradative enzyme activation lead to cartilage damage and bone changes, which in the end can progress to joint space narrowing and ankyloses.

Traditionally, CR is used to assess the bone (osteopenia, periostitis, erosions), joint (narrowing, effusion, synovitis), and growth (malalignment, length discrepancy) abnormalities in JIA. Recently introduced pediatric scoring systems have increased the value of CR in JIA. However, as CR can be normal or only show minor changes early in the disease and outcome and prognosis correlate with early initiation of treatment, there is an increasing emphasis on the detection of articular inflammation before radiographically detectable changes occur. This is much better evaluated with US and MRI. Several studies have shown that US outperforms clinical evaluation in the identification of synovitis and in distinguishing joint disease from tenosynovitis. US appears to be particularly useful in the evaluation of deeper joints, such as the hip, and smaller complex joints, such as the ankle, midfoot, and wrist. Imaging findings on US include synovial hypertrophy (uniform, nodular, fibrous stands, villi), joint effusion, and increased color Doppler signal. It is good to realize that isolated joint fluid is not specific for articular inflammation because joint effusion is common in healthy children; e.g., it is present in up to $60 \%$ of knees and often seen in at least one joint of the wrist. Tenosynovitis is another imaging finding that is frequently seen in JIA, characterized

Table 20.2 Subtypes of juvenile idiopathic arthritis (JIA) according to the Revised Criteria of the International League of Associations for Rheumatology [24]

\begin{tabular}{|c|c|c|c|c|}
\hline Subtype & Definition & $\begin{array}{l}\text { Frequency } \\
(\%)\end{array}$ & Age at onset & Sex ratio \\
\hline Systemic arthritis & $\begin{array}{l}\text { Presence of arthritis accompanied or preceded by daily fever of at least } 2 \text {-week } \\
\text { duration plus at least one of the following: typical evanescent erythematous rash, } \\
\text { hepatomegaly or splenomegaly, generalized lymphadenopathy, or serositis }\end{array}$ & $4-17 \%$ & $\begin{array}{l}\text { Throughout } \\
\text { childhood }\end{array}$ & $\mathrm{F}=\mathrm{M}$ \\
\hline Oligoarthritis & $\begin{array}{l}\leq 4 \text { joints affected during the first } 6 \text { months of disease, subtypes: } \\
\quad-\text { Persistent: confined to } \leq 4 \text { joints throughout the disease course } \\
-\quad \text { Extended: extension to }>4 \text { joints after first } 6 \text { months of the disease }\end{array}$ & $27-56 \%$ & $\begin{array}{l}\text { Early } \\
\text { childhood }\end{array}$ & $\mathrm{F} \gg \gg \mathrm{M}$ \\
\hline $\begin{array}{l}\text { Polyarthritis, RF } \\
\text { negative }\end{array}$ & $\geq 5$ joints affected during first 6 months of disease and absence of IgM RF & $11-28 \%$ & $\begin{array}{l}\text { Biphasic: } \\
2-4 \text { years } \\
6-12 \text { years }\end{array}$ & $\mathrm{F}>\mathrm{M}$ \\
\hline $\begin{array}{l}\text { Polyarthritis, RF } \\
\text { positive }\end{array}$ & $\begin{array}{l}\geq 5 \text { joints affected during first } 6 \text { months of disease and presence of IgM RF on at } \\
\text { least two occasions more than } 3 \text { months apart }\end{array}$ & $2-7 \%$ & $\begin{array}{l}\text { Late childhood } \\
\text { or adolescence }\end{array}$ & $\mathrm{F}>>\mathrm{M}$ \\
\hline Psoriatic arthritis & $\begin{array}{l}\text { Combination of arthritis and typical psoriatic rash or (if rash is absent) presence } \\
\text { of arthritis and any two of the following: family history of psoriasis in a } \\
\text { first-degree relative, dactylitis, or nail pitting }\end{array}$ & $2-11 \%$ & $\begin{array}{l}\text { Biphasic: } \\
\text { 2-4 years } \\
9-11 \text { years }\end{array}$ & $\mathrm{F}>\mathrm{M}$ \\
\hline $\begin{array}{l}\text { Enthesitis-related } \\
\text { arthritis }\end{array}$ & $\begin{array}{l}\text { Enthesitis and/or arthritis; most common sites of enthesitis are calcaneal insertion } \\
\text { of the Achilles tendon, plantar fascia, and tarsal area; arthritis commonly affects } \\
\text { joints of lower extremities; sacroiliac involvement and lumbosacral spine may } \\
\text { occur; most children are HLA-B27 positive or have family history of HLA-B27- } \\
\text { associated disease }\end{array}$ & $3-11 \%$ & $\begin{array}{l}\text { Early } \\
\text { childhood or } \\
\text { adolescence }\end{array}$ & $M \gg F$ \\
\hline $\begin{array}{l}\text { Undifferentiated } \\
\text { arthritis }\end{array}$ & $\begin{array}{l}\text { Arthritis that either does not fulfill any of the subtypes or can fulfill } \geq 2 \text { other } \\
\text { subtypes }\end{array}$ & $11-21 \%$ & NA & NA \\
\hline
\end{tabular}

$R F$ rheuma factor, $N A$ not available 
by abnormal hypoechoic or anechoic thickening of the tendon sheath with or without accumulation of fluid. Color Doppler signal can be present but is not required for diagnosis. Tenosynovitis is most commonly seen around the ankle joint and in the extensor compartment of the wrist. Other US findings include increased color Doppler signal in the epiphyseal cartilage, decreased thickness of the joint cartilage, periarticular erosions, and enthesopathy. On MRI involved joints will show synovial thickening, joint effusion, and/or synovial enhancement on contrast-enhanced sequences. Furthermore, MRI can demonstrate signs of tenosynovitis and enthesitis, cartilage damage and bony erosions (epiphyseal), bone marrow edema, and increased vascularity of epiphyseal cartilage. It is good to realize that the widespread adoption of US and MRI in the diagnosis and followup of JIA requires a good understanding of the imaging findings and its clinical implications, as well as the development of validated pediatric joint assessment protocols. Furthermore, a substantial number of healthy children appear to have "abnormalities" in one or more joints, including bone marrow edema, joint effusion, erosion-like lesions, and even synovial thickening and signs of tenosynovitis. That is why current research is focusing on differentiating pathologic bony, cartilage, and other articular abnormalities from normal developmental variants.

\section{Key Point}

- Knowledge of the many normal variants in the pediatric musculoskeletal system that can simulate disease is essential to be able to differentiate them from pathologic bony, cartilage, and articular abnormalities.

\subsection{Neoplastic Diseases}

\subsubsection{Bone Tumors $[28,29]$}

The vast majority of pediatric bone tumors are benign and include osteochondroma, nonossifying fibroma, unicameral or simple bone cyst (UBC), aneurysmal bone cyst (ABC), and osteoid osteoma. They are most frequently found between 5 and 25 years of age, often incidentally (e.g., after minor trauma). Benign bone tumors usually do not require treatment or follow-up, although some might become symptomatic and may require (surgical) intervention (e.g., pedunculated osteochondroma and osteoid osteoma). When patients are symptomatic, they most commonly present with pain that is mild, only activity-related, and nonprogressive. Nonprogressive nocturnal pain reliably relieved with aspirin or nonsteroidal anti-inflammatory drugs is typical of an oste- oid osteoma. Sometimes the presenting symptom is a pathologic fracture (e.g., in UBC).

Malignant bone tumors constitute around $6 \%$ of all childhood cancer in patients under the age of 20 years and are the seventh most common type of tumors in children. Approximately two-thirds of these are osteosarcomas, with the remainder largely belonging to the Ewing sarcoma family of bone tumors. Malignant bone tumors occur mainly during adolescence and young adulthood. Patients with malignant bone tumors nearly always present with complaints of deep, achy, and unrelenting pain and swelling. A pathologic fracture, although uncommon, can also be a presenting feature in a minority of malignant bone tumors.

Imaging studies are essential for diagnosis, planning biopsy (if indicated), and follow-up during treatment. They can aid in differentiating a benign from malignant lesion by providing essential information regarding its nature, size, anatomic location, and effect on surrounding bone or soft tissues. Furthermore, involvement of adjacent joints and neurovascular structures can be assessed which is particularly important when surgical intervention is considered or indicated. CR should always be obtained before advanced imaging is performed as it is very helpful in establishing a first differential diagnosis. Imaging findings that can be assessed on $\mathrm{CR}$ include anatomic location of the lesion and position within a given bone; whether the tumor is radiolucent or radiodense or has a mixed appearance; and what the tumor's effect is on the bone and the bone's effect on the tumor. Benign bone tumors usually have sharply demarcated margins with a narrow transition zone with a geographic pattern of bone destruction with smooth and uninterrupted periosteal reaction. Malignant bone tumors, on the other hand, usually have poorly defined margins with a wide zone of transition suggesting a fast-growing lesion and a permeative or motheaten pattern of bone destruction with a malignant (sunburst or onion skin-like) periosteal reaction (Fig. 20.5). In most benign bone tumors, CR will suffice and no further imaging is needed. Computed tomography has a limited role to play in pediatric patients and must be used only when a real benefit is expected (e.g., for diagnosis and/or treatment of osteoid osteoma or in CT-guided biopsies). MRI is the imaging modality of choice, in particular in (suspected) malignant bone tumors, to define tumoral extension within the bone and in the soft tissues, involvement of adjacent joints and neurovascular structures, and presence/absence of skip lesions. It should be performed before a (needle) biopsy is performed as this can induce edema or hematoma, which will impact the image quality and interpretation. Furthermore, MRI is used as follow-up during chemotherapy and for preoperative planning after chemotherapy. The MRI protocol should include at least coronal T1-, axial T2-, 3D T1-weighted contrast-enhanced fat saturation sequences, as well as DWI. A T1- or STIR-weighted sequence in the sagittal or coronal 


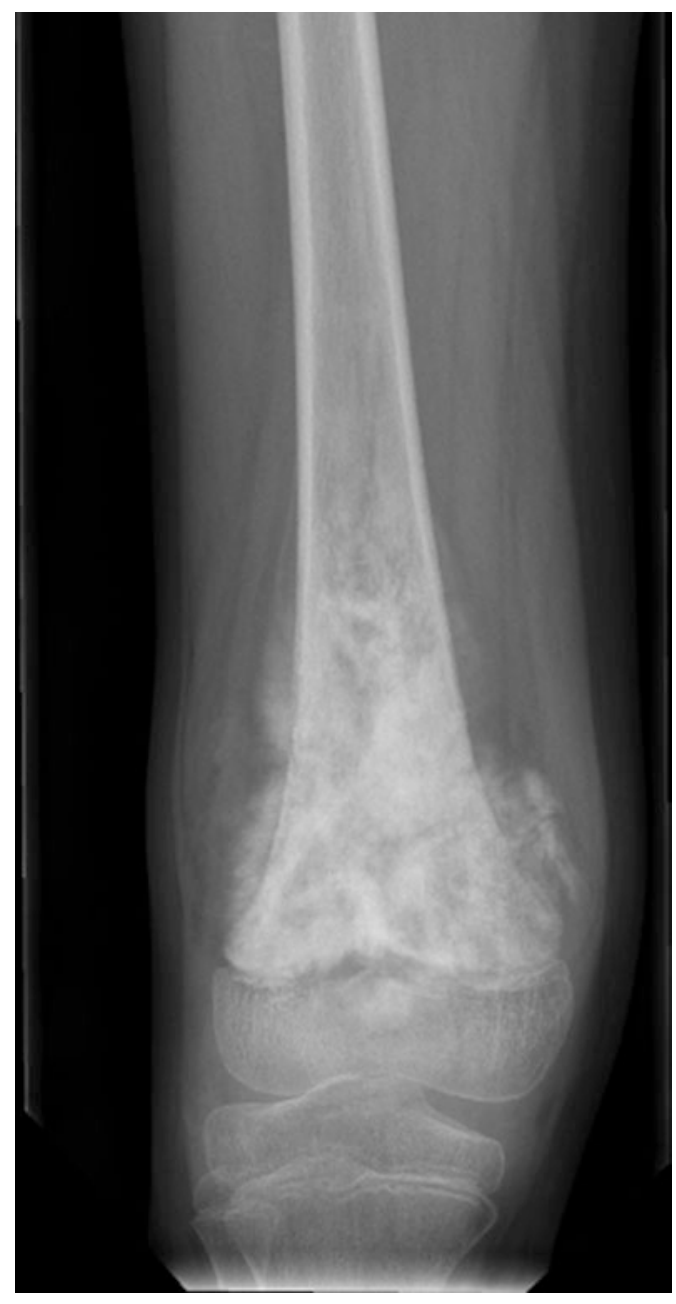

Fig. 20.5 X-ray of the right distal femur illustrating a malignant periosteal reaction (sunburst aspect with Codman's triangles) in a 9-yearold child with osteosarcoma. Furthermore, a permeative sclerotic mass is seen with osteoid formation and extension into the adjacent soft tissues

plane covering the entire bone should be added to look for skip metastases metastases. 18F-FDG PET/CT has also been used to assess metastatic disease as well as the response to neoadjuvant chemotherapy, but its use depends on the type of malignant bone tumor and/or treatment protocol.

\subsubsection{Soft Tissue tumors [30-33]}

As in pediatric bone tumors, the overwhelming majority of soft tissue tumors in children is benign. The most common benign soft tissue tumors are the vascular tumors and malformations, followed by lipomas, pseudotumors, fibrohistio- cytic tumors, and peripheral nerve sheath tumors. The presenting symptom is usually a bump or fullness which can be symptomatic or entirely painless. In general, benign soft tissue tumors are less than $4 \mathrm{~cm}$ in diameter. Malignant soft tissue tumors are rare, accounting for only $1 \%$ of all soft tissue tumors in children. Soft tissue sarcomas constitute $7 \%$ of all the childhood cancers in patients under the age of 20 years and are the sixth most common type of tumor in children. Rhabdomyosarcoma (RMS) represents $50 \%$ of all soft tissue sarcomas. The most frequent non-RMS malignant tumors are peripheral primitive neuroectodermal/Ewing sarcoma family tumors (pPNET/Ew) (19\%), malignant peripheral nerve sheath tumors $(6.5 \%)$, infantile fibrosarcomas $(6 \%)$, and synovial sarcomas (SNVS) (5\%). Age of the patient, site of the lesion, clinical history, and especially chronicity of the lesion are cornerstones in the differential diagnosis. Furthermore, predisposing diseases and/or syndromes must be taken into account (e.g., neurofibromatosis, Li-Fraumeni syndrome). Clinical criteria that are suspicious of a malignant soft tissue tumor include a size greater than $5 \mathrm{~cm}$, increasing size over time, pain, and a deep location of the tumor.

US is the imaging modality of first choice, especially for small lesions. Imaging features to look for include size, regular/sharp versus irregular margins, solid versus cystic components, echogenicity, fluid-fluid levels, calcifications, and vascularity on color Doppler imaging. CR can be used to detect and characterize calcifications and involvement of the adjacent bones (scalloping and erosion versus a destructive/ invasive appearance). If a definitive diagnosis cannot be made based on the US and/or CR appearance, MRI is the imaging modality of choice to further explore the lesion. As in bone tumors, this should be done prior to biopsy or surgery to accurately delineate tumor composition and extension, as well as its relation to adjacent anatomical structures such as bone, joints, and neurovascular bundle. The MRI protocol should at least include T1- and T2-weighted sequences without/with fat saturation and contrast-enhanced T1-weighted sequences. The addition of DWI is recommended but not mandatory, as its role in distinguishing benign from malignant tumors and evaluation of response to therapy has not yet been conclusively demonstrated.

\section{Key Point}

- The vast majority of bone and soft tissue tumors in children is benign. 


\subsection{Concluding Remarks}

Imaging plays an essential role in the diagnosis and followup of musculoskeletal diseases in children. The radiological evaluation is challenging because of anatomical and physiological differences between the pediatric and adult musculoskeletal system, as well as the great variety of diseases and its typical presentation in children. Knowledge of these differences and the optimal choice of imaging techniques are essential to prevent any unnecessary delay in diagnosing pediatric musculoskeletal diseases.

\section{Take Home Messages}

- The clinical and radiological presentation of musculoskeletal diseases in children differs in many ways from adults, in particular related to anatomical and physiological differences of the musculoskeletal system.

- There are many normal variants in the developing skeleton that may simulate pediatric musculoskeletal disease.

- Knowledge of these differences and of the optimal choice of imaging techniques is essential for an adequate diagnosis of musculoskeletal diseases in children.

- Ultrasound (US) and conventional radiography (CR) are the imaging modalities of first choice in most pediatric musculoskeletal diseases.

\section{References}

1. Perry DC, Bruce C. Evaluating the child who presents with an acute limp. BMJ. 2010;341:c4250.

2. Zucker EJ, Lee EY, Restrepo R, Eisenberg RL. Hip disorders in children. AJR. 2013;201:W776-96.

3. Silva MS, Fernandes ARC, Cardoso FN, Longo CH, Aihara AY. Radiography, CT, and MRI of hip and lower limb disorders in children and adolescents. Radiographics. 2019;39:779-94.

4. Parcels BW. Pediatric hip and pelvis. Pediatr Clin N Am. 2020;67:139-52.

5. Graf R. Hip sonography. Diagnosis and management of infant hip dysplasia. Berlin: Springer; 2006.

6. Robben SGF, Smithuis R. Developmental dysplasia of the hip - Ultrasound (2017) Radiology Assistant. 2017. https://radiologyassistant.nl/pediatrics/hip/developmental-dysplasia-of-thehip-ultrasound.

7. Beek FJ, Nievelstein RJ, Pruijs HE, de Jong PA, Sakkers RJ. Transinguinal sonographic determination of the position of the femoral head after reposition and follow-up in a spica cast. Pediatr Radiol. 2010;40(11):1794-9.

8. Dillman JR, Hernandez RJ. MRI of Legg-Calvé-Perthes disease. AJR. 2009;193:1394-407.
9. Dwek JR. The hip: MR imaging of uniquely pediatric disorders. Magn Reson Imaging Clin N Am. 2009;17:509-20.

10. Shah H. Perthes disease: evaluation and management. Orthop Clin N Am. 2014;45:87-97.

11. Cardinal E, Bureau N, Aubin B, et al. Role of ultrasound in musculoskeletal infection. Radiol Clin N Am. 2001;39:191-201.

12. Karmazyn B. Imaging approach to acute hematogenous osteomyelitis in children: an update. Semin Ultrasound CT MRI. 2010;31:100-6.

13. Kan JH, Young RS, Yu C, et al. Clinical impact of gadolinium in the MRI diagnosis of musculoskeletal infection in children. Pediatr Radiol. 2010;40:1197-205.

14. Jaramillo D. Infection: musculoskeletal. Pediatr Radiol. 2011;41(Suppl 1):S127-34.

15. van Schuppen J, van Doorn MM, van Rijn RR. Childhood osteomyelitis: imaging characteristics. Insights Imaging. 2012;3:519-33.

16. Montgomery NI, Rosenfeld S. Pediatric osteoarticular infection update. J Pediatr Orthop. 2015;35:74-81.

17. De Moraes Barros Fucs PM, Meves R, Henzo Yamada H. Spinal infections in children: a review. Int Orthopaed (SICOT). 2012;36:387-95.

18. Nguyen JC, Lee KS, Thapa MM, Rosas HG. US evaluation of juvenile idiopathic arthritis and osteoarticular infection. Radiographics. 2017;37:1181-201.

19. Struk DW, Munk PL, Lee MJ, et al. Imaging of soft tissue infections. Radiol Clin N Am. 2001;39:277-303.

20. Trusen A, Beissert M, Schultz G, et al. Ultrasound and MRI features of pyomyositis in children. Eur Radiol. 2003;13:1050-5.

21. Khanna G, Sato TS, Ferguson P. Imaging of chronic recurrent multifocal osteomyelitis. Radiographics. 2009;29:1159-77.

22. Falip C, Alison M, Bourty N, et al. Chronic recurrent multifocal osteomyelitis (CRMO): a longitudinal case series review. Pediatr Radiol. 2013;43:355-75.

23. Zhao Y, Ferguson PJ. Chronic nonbacterial osteomyelitis and chronic recurrent multifocal osteomyelitis in children. Pediatr Clin N Am. 2018;65:783-800.

24. Damasio MB, Malattia C, Martini A, Tomà P. Synovial and inflammatory diseases in childhood: role of new imaging modalities in the assessment of patients with juvenile idiopathic arthritis. Pediatr Radiol. 2010;40:985-98.

25. Chauvin NA, Doria AS. Ultrasound imaging of synovial inflammation in juvenile idiopathic arthritis. Pediatr Radiol. 2017;47:1160-70.

26. Dimitriou C, Boitsios G, Badot V. Imaging of juvenile idiopathic arthritis. Radiol Clin N Am. 2017;55(5):1071-83.

27. Malattia C, Rinaldi M, Martini A. The role of imaging in juvenile idiopathic arthritis. Expert Rev Clin Immunol. 2018;14(8):681-94.

28. Shah JN, Cohen HL, Choudhri AF, et al. Pediatric benign bone tumors: what does the radiologist need to know? Radiographics. 2017;37:1001-2.

29. Singla A, Geller DS. Musculoskeletal tumors. Pediatr Clin N Am. 2020;67:227-45.

30. Navarro OM, Laffan EE, Ngan BY. Pediatric soft-tissue tumors and pseudotumors: MR imaging features with pathologic correlation (Part 1). Radiographics. 2009;29:887-906.

31. Laffan EE, Ngan BY, Navarro OM. Pediatric soft-tissue tumors and pseudotumors: MR imaging features with pathologic correlation (Part 2). Radiographics. 2009;29(4):e36.

32. Brisse HJ, Orbach D, Klijanienko J. Soft tissue tumours: imaging strategy. Pediatr Radiol. 2010;40:1019-28.

33. Inarejos Clemente EJ, Navallas M, Barber Martínez de la Torre I, et al. MRI of rhabdomyosarcoma and other soft-tissue sarcomas in children. Radiographics. 2020;40:791-814. 
Open Access This chapter is licensed under the terms of the Creative Commons Attribution 4.0 International License (http://creativecommons. org/licenses/by/4.0/), which permits use, sharing, adaptation, distribution and reproduction in any medium or format, as long as you give appropriate credit to the original author(s) and the source, provide a link to the Creative Commons license and indicate if changes were made.

The images or other third party material in this chapter are included in the chapter's Creative Commons license, unless indicated otherwise in a credit line to the material. If material is not included in the chapter's Creative Commons license and your intended use is not permitted by statutory regulation or exceeds the permitted use, you will need to obtain permission directly from the copyright holder.

(c) (i) 